\title{
CONSTRUCCIÓN DE SENTIDOS SOBRE LA ESCUELA EN JÓVENES Y ADOLESCENTES: UN ABORDAJE A PARTIR DEL ANÁLISIS DE LA CONFIGURACIÓN ACTUAL DEL MERCADO LABORAL (1)
}

\author{
Franco Nicolás Carbonari (Universidad Tecnológica Nacional)* \\ orko215@hotmail.com \\ Julia Marchetti (Universidad de Concepción del Uruguay)** \\ yulilaleprosa@hotmail.com
}

Recibido: 31/07/2012 Aceptado: 2/09/2012

\begin{abstract}
Resumen
El presente artículo tiene como fin rastrear aspectos que den cuenta de los elementos que influyen en la construcción de sentidos que los jóvenes le dan a la escuela media en el paso por sus vidas. Siguiendo este objetivo; nos proponemos observar a partir de un análisis comparativo a nivel socio-histórico en el contexto argentino, si hay una reconfiguración de sentidos que se enlace con los cambios experimentados por mercado laboral. En este sentido, comenzamos a indagar a partir de la descripción de una realidad social donde el trabajo ha dejado de ser el eje organizador de la vida de los sujetos en la década de los 90', y como la escuela se ubicó ante esa realidad en la representación que los jóvenes tienen de ella. Finalmente, arribamos a pensar como pueden ser reconfigurados o no estos sentidos en función de entender el funcionamiento del mercado laboral en la actualidad.
\end{abstract}

\section{Palabras clave:}

Trabajo - Escuela - Sentidos - Jóvenes - Trayectorias.

* Profesor en Ciencias de la Educación. Universidad Nacional de Rosario. Becario en la Universidad Tecnológica Nacional. Doctorando en Educación. Universidad Nacional de Entre Ríos.

** Lic. en Sociología. Universidad de Buenos Aires. Cursando la Especialización en Gestión Estratégica de Organizaciones Públicas. Universidad Nacional De Rosario. 


\begin{abstract}
This article want to trace aspects that account for the elements that influence the construction of meanings that young people give the middle school as a step for their lives. Following this objetive, we propose to observe from a comparative socio-historical analysis in Argentine context, if there is a meanings reconfiguration that link the changes in the labor market. In this sense, we began to inquire from the description of a social reality where work has ceased to be the principal element in the life of the subjects from the 90's, and how the school was located at that reality in relation to the representation that young people have it. Finally, we come to think how they can reconfigured or not these senses in order to understand the functioning of the labor marked today.
\end{abstract}

\title{
Key words:
}

Work - School - Senses - Younges - Paths.

\section{Introducción}

La propuesta principal del presente artículo es poner en diálogo los elementos mencionados en el título en pos de indagar acerca de las nuevas configuraciones de relación entre ellos.

Nuestro recorrido se centra en desarrollar una descripción de la escuela como una institución atravesada por una crisis de sentidos enmarcada en un contexto donde el resto de las instituciones de la sociedad se encontrarían experimentando una situación similar. En este sentido, a través de distintos autores, comenzamos planteando un acercamiento a las modificaciones sucedidas en nuestro país con más intensidad en la década de los 90 .

Modificaciones que a partir del cambio en el patrón de acumulación económica, repercutían en todos los estamentos sociales, incluidos los sentidos construidos por la sociedad. En esta línea de análisis es que incluimos la morfología que a grandes rasgos presentaba el mercado laboral, destacando entre otras características la de la desocupación, la precarización y la flexibilización.

Este desarrollo de características predominantemente sociológicas, encuentra su sustento en una hipótesis muy lineal. Puntualmente, la idea que daba vueltas se podría describir de la siguiente manera: Los procesos de desocupación, precarización, flexibilización que se producen muy fuertemente en la década de los 90 , vivenciados por los padres de muchos de los jóvenes que hoy deberían escolarizarse, inciden muy fuertemente en la valorización que esos jóvenes realizan sobre la escuela. Y teniendo en cuenta lo traumático (2) de dichas experiencias sufridas por los sujetos, sobre todo de sectores populares, la valoración que hagan de la escuela media sería más que nada, y expresado burdamente, negativa. Reconociendo en tal situación los motivos del abandono escolar de estos jóvenes, desde una lectura pragmática, la mirada estaba puesta en cierto valor instrumental con que los sujetos significaban la escuela. 
De alguna manera, se intenta direccionar el análisis del presente trabajo hacia el objetivo de rastrear aspectos de la estructura social que den cuenta de una potencial construcción de sentidos que los jóvenes le dan a la escuela media en el paso por sus vidas. Siguiendo este objetivo; nos proponemos indagar a partir de un análisis comparativo a nivel socio-histórico del contexto argentino, si hay una reconfiguración de sentidos que se enlace con los cambios experimentados por el mercado laboral. Esto es, comenzamos a describir una realidad social donde el trabajo ha dejado de ser el eje organizador de la vida de los sujetos en la década de los 90', y como la escuela se ubica ante esa realidad en la representación que los jóvenes tienen de ella. Y arribamos a pensar cómo se van reconfigurando o no estos sentidos en función de entender el funcionamiento del mercado laboral actual.

Basándonos básicamente en planteos como los F. Dubet (2006) en relación a la decadencia de lo que el denominaría programa institucional, programa que incluía a la institución escolar como uno de sus componentes, es que intentamos pensar la construcción de sentido sobre la escuela en relación a la configuración del mercado laboral. Dubet va a plantear:

La decadencia del programa institucional proviene de la exacerbación de sus contradicciones latentes [...] A causa de la creciente complejidad de la división del trabajo, de la pluralidad y de la débil coherencia de los roles que deben asumir los individuos, se abre la distancia entre las motivaciones y las acciones esperadas (2006: 64).

Es decir, la creciente complejidad del trabajo como premisa que quiebra el principio que establecía una línea de continuidad entre socialización y subjetivación, y entre la disciplina y la autonomía que proveía como efecto el programa institucional:

Esa cadena milagrosa (línea de continuidad entre socialización y subjetivación) ya no funciona o, para optar por una fórmula más prudente, los actores ya no creen en ella, porque la correspondencia entre las dimensiones subjetivas de la acción y las dimensiones objetivas del estatuto, se debilitaron (Dubet, 2006: 81).

Ahora bien, si se realiza la aclaración sobre el carácter comparativo a nivel socio-histórico que presentará el siguiente artículo, la misma se basa en tratar de evitar los potenciales riesgos de pensar la relación entre los sentidos construidos sobre la escuela y la configuración del mercado laboral en nuestro país recurriendo a caracterizaciones donde lo que refleja el paisaje de dicho mercado son las situaciones de desocupación, precarización, flexibilización (situaciones generalizadas sin duda en lo que fue la década del '90 y una parte de la primer década del SXXI). Uno de los elementos que argumentan la necesidad de replantearnos la configuración del mercado laboral se representa en el cuadro 
número 1. En el mismo se grafican los niveles de la tasa de desocupación y subocupación en el período comprendido entre el primer trimestre del 2003 y el primer trimestre de 2012.

\section{Cuadro $N^{\circ} 1$ (3)}

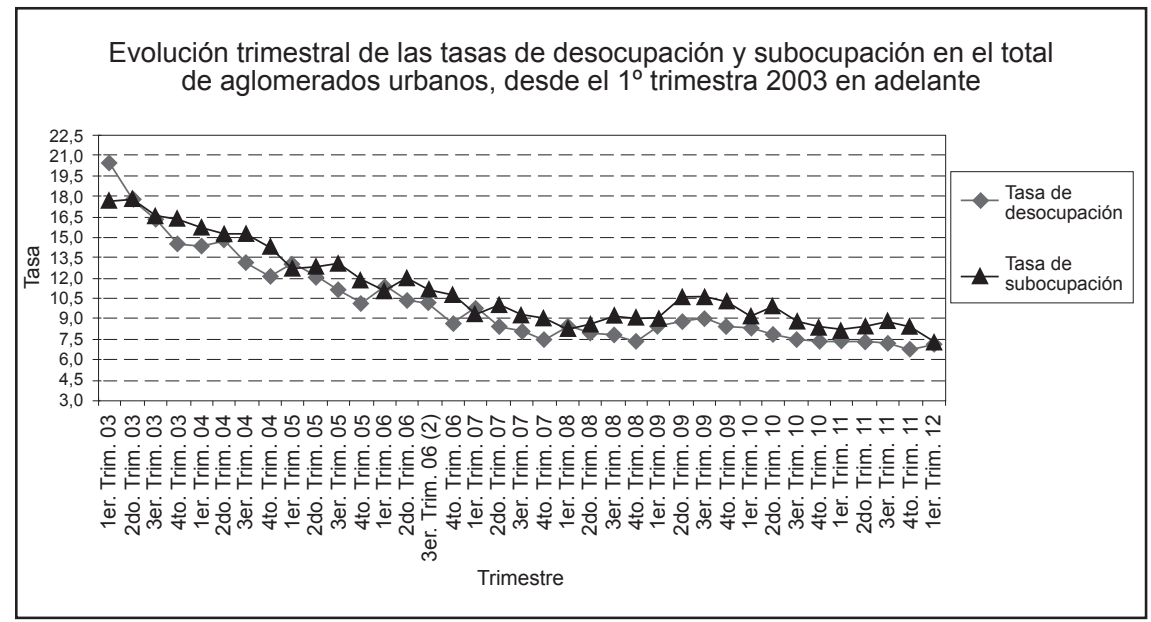

En relación a la potencial miopía en la que podemos incurrir al no anclar nuestros análisis en una nueva configuración del mercado laboral, Palomino (2010) expresará:

La reflexión sobre estos temas sigue de cierta manera retrasada con respecto a la evolución real, generando un fenómeno de "disonancia cognitiva", en el sentido de la persistencia en la percepción de ciertas "figuras", cuando el "fondo" del panorama registra un cambio considerable y reclama, sin dudas, un cambio de enfoque. (p. 222)

En definitiva, a partir del presente análisis solo se intentará ampliar la mirada sobre un fenómeno que por su complejidad ni si quiera el comienzo de su estudio se agota en este artículo.

Tal vez, otro de los motores que ha propulsado el presente artículo es la cuestión del abandono escolar. Si bien no aparece como eje central de nuestro análisis, si sea uno de los hechos que nos moviliza a desandar caminos varios como el reflejado en estas líneas. Sabemos que el mismo debe ser considerado solo como uno de los aspectos o vías entre otros varios para pensar dicha cuestión. Por lo tanto, las reflexiones que surjan del presente acercamiento no derivarán más que en aristas que ofrezcan indicios, desde un punto de vista ubicado en la estructura social, de ciertas motivaciones a las cuáles podemos 
llamar sentidos que pueden llegar a vivenciar determinados sujetos. Y si bien nuestro análisis apunta a delinear la construcción de sentidos en relación a la escuela media, lo que pueda llegar a surgir del mismo deberá ser considerado como un elemento más desde donde pensar la relación de jóvenes y adolescentes con la escuela media, simplemente, y no más que eso, un elemento entre otros.

\section{Acerca de la construcción de sentidos sobre la escuela media.}

En esta instancia vemos al mercado laboral como un elemento que dentro de la estructura de la sociedad Argentina coadyuva en la conformación de sentidos históricamente dados, y además de ser un portador de gran dimensión en su condición de organizador social.

En este sentido, Pogliaghi (2010) reconocerá la existencia de numerosos estudios que han verificado que en épocas anteriores, la inserción de los individuos en el mercado de trabajo a partir de empleos estables y protegidos era uno de los principales, sino el más importante medio de integración social. Por otro lado, colocará en las condiciones del mercado laboral una pieza fundamental a la hora de pensar el ejercicio pleno de la ciudadanía.

En este marco, es que pensar los sentidos sobre la escuela no debe ser un ejercicio que sesgue su mirada en aspectos coyunturales o contingentes abocados únicamente a pensar lo que sucede dentro de las escuelas, o de forma aislada indagar sobre lo que vivencian los sujetos que la transitan, o los que nunca la transitaron, o los que en un momento lo hicieron y luego han dejado de hacerlo.

Es decir, pensar los sentidos construidos alrededor de la escuela media implica remontarnos a la aparición de dicha institución en nuestro país y reflexionar sobre los lazos simbólicos establecidos entre ésta y la población de manera que el recorrido de cuenta sobre todo de ciertos aspectos considerados valiosos en el proceso de construcción de sentidos.

De esta manera, podemos decir que más allá de las profundas modificaciones producidas a lo largo de la historia en el sistema educativo argentino la institución escolar ha representado, con más intensidad en determinados momentos que en otros, cierta alianza con la población, especialmente con los sectores populares quienes veían en dicha institución una promesa de promoción económica y social (Tiramonti, 2005; Braslavky, 1985). De esta manera, y a partir de la mediación del Estado como garante de canalizar estas expectativas ampliando la oferta escolar (Tiramonti, 2005), y a pesar de que la escuela con su lógica "igualadora" ejercía cierto grado de violencia simbólica sobre sectores que no adecuaban sus hábitos y prácticas a las requeridas por el ámbito escolar, el discurso igualador disponía de cierta eficacia que provocaba un grado de consenso entre distintas fracciones de clases sociales, tal proceso fue ubicando a la escuela en un lugar de autoridad indiscutible. Al hablar de 
los sentidos construidos alrededor de esta relación, Zakï Laïdi (En Tedesco, 2005) va a plantear:

La expansión de la escuela implicaba brindar a los sectores populares un conjunto de herramientas que permitían un desarrollo personal y social muy significativo [...] La fertilidad socializadora de este proyecto radicaba en que era portador de un sentido (p. 5).

Como se señaló anteriormente, la eficacia simbólica (4) expresada en el tipo de alianza establecida entre la escuela y la población estuvo fuertemente marcada por el ascenso en la escala social, tanto material como simbólico, que habilitaba el paso por la escuela.

Para describir este escenario inspirado por la modernidad, Francois Dubet (2006) utilizará la expresión programa institucional, describiendo al mismo como el "[...] proceso social que transforma valores y principios en acción y en subjetividad por el sesgo de un trabajo profesional específico y organizado [...] Como un tipo de relación social y de trabajo institucional sobre los otros" (pp. 32 - 61).

Según este autor el programa institucional posibilitó el afianzamiento del individuo en tanto actor social y sujeto, considerando junto a otros autores, que este proceso de individualización encuentra su origen en la división del trabajo. Sobre dicho fenómeno expresará: "Ella hará emerger la noción de rol que implica una separación entre los actores y una promoción legítima de sus intereses individuales, una finalización de un enclave en las comunidades, cierta movilidad y una autonomía relativa de las conciencias" (Dubet, 2006: 45).

De esta manera se enumeran, según Dubet, algunas de las consecuencias producidas por la división del trabajo a fines del SXVIII, que por lo pronto establecía un quiebre con una sociedad tradicional en la cuál el individuo se encontraba totalmente devorado por la colectividad (Dubet, 2006).

Recuperando la idea inicial que ubica al Estado como garante de la oferta educativa; agregamos que el mismo jugó el rol de garante, ordenador, administrador, y actor clave en el funcionamiento de estas instituciones, ocupando un lugar fundamental a la hora de lograr resultados en la idea de construir sentidos que garanticen el orden social a partir de reglas disciplinadoras:

La organización estado céntrica de la sociedad correspondió a la conformación de la sociedad industrial y del entramado institucional propio de esta etapa del desarrollo capitalista: la fabrica, la familia, la escuela, la clase social, las instituciones de representación política y sectorial constituyeron marcos institucionales que regulaban y contenían la existencia de los individuos hasta avanzado el siglo XX. Se trataba de un complejo institucional cuya eficacia resultaba de la complementariedad de su acción y su común referencia al Estado Nacional (Tiramonti, 2005, p. 17). 
Ahora bien, pensar los sentidos construidos en este momento histórico por jóvenes y adolescentes en relación a la escuela, implica considerar la decadencia del programa institucional como la decadencia de un fenómeno que afecta nuestra concepción misma de la vida social y de la acción, y no sólo al trabajo sobre los otros. "La decadencia del programa institucional proviene de la exacerbación de sus contradicciones latentes, cuando ya no posee la capacidad ideológica para borrarlas [...] la fuerza para superar paradojas por el don de su magia" (Dubet, 2006, p. 64).

En esta misma línea Silvia Duschatzky (1999: 18) va a plantear: "Pensar los sentidos sobre la escuela [...] está en la construcción interpretativa que supone inscribir lo dicho en un contexto más amplio de significación". Es aquí donde radica la importancia de reflexionar sobre la morfología del mercado laboral y su incidencia en la construcción de sentidos.

\section{Mercado laboral en los '90.}

\section{El trabajo y la escuela en la era del declive}

Entendemos, como plantea Gentili (1997), que el capitalismo atraviesa a lo largo de toda su historia una serie de procesos de cambio y ruptura tanto cuali como cuantitativos de tipo estructural. A su vez, estos procesos son permanentes y cíclicos, e involucran a todas las esferas de práctica social. Es decir, que toda dinámica de crisis "[...] al mismo tiempo en que expresa la necesidad de resolver las contradicciones inherentes a este tipo de sociedades, constituye una nueva instancia generadora de contradicciones que definirán la idiosincrasia del período que se inicia luego de cada ruptura" (Citado en Castillo, 2006: 10). Dicho proceso no cuestiona la naturaleza y el carácter del modo de producción, sino que imprime a este último una renovada morfología de sentido transitorio y relativamente estable que se reproducirá hasta el surgimiento de una nueva crisis.

Teniendo en cuenta la dinámica del modo de producción capitalista, nos atrevemos a expresar que la realidad de nuestro país nos encuentra frente a un momento donde el predominio de los sectores que se posicionan como hegemónicos a partir de los cambios en la estructura económica producidos a mediados de la década de los 70 , no goza de total libertad para ejercer dicha hegemonía. En este marco, el presente apartado tiene la intención de llevar a cabo un recorrido que de cuenta de las características que se supo imprimirle al modelo de relaciones laborales durante la etapa en la que el modo de producción capitalista con predominio del sector financiero avanzaba sin escollos sobre todos los estamentos de la sociedad.

A partir de la década de los 70' dicha etapa del modelo capitalista experimenta un salto cualitativo en la expansión de sus fuerzas productivas a escala planetaria. Se genera un aceleramiento del crecimiento del sistema productivo. 
Esto es: reemplazo de fuerza de trabajo por capital, que se combina con el incremento persistente de los ritmos del proceso de trabajo, donde un volumen cada vez más pequeño de fuerza de trabajo, produce un volumen cada vez mayor de valor. Es decir, cada vez menos trabajadores son necesarios para producir una mayor cantidad de mercancías (Carbonari, 2009).

Igualmente, el auge de esta forma del modo de producción capitalista en nuestro país se observa durante la década de los '90. Para el periodo 1998-9, ya más del $50 \%$ de la estructura económico social se encuentra bajo dominio del capital financiero transnacional.

Observamos como este proceso que comienza en la década de los 70', empieza a reconfigurar el mapa del mercado laboral. Exigiendo -entre otros insumos-, una fuerza de trabajo flexible para la actividad económica-productiva. Esta flexibilidad, se necesita en la medida en que se requiera de una mayor disponibilidad de trabajo para su rotación, capacitación o eventual reemplazo. (Carbonari; 2009).

Por otra parte, durante este período, el mercado laboral junto a la estructura social sufrió fuertes procesos de segmentación entre los trabajadores integrados al mercado formal y las capas crecientes de trabajadores informales y precarizados. El cuentapropismo y el empleo informal adquieren una nueva centralidad social y productiva. Los trabajadores que ofrecen su fuerza de trabajo al mercado informal están sujetos a diferentes mecanismos de precarización e inserción inestable que los asocia a crecientes niveles de marginación social (Catalano y Novick, 1996).

Se va a decir que ya hacia fines de 1989 se perfila la articulación de un programa de estabilización con reformas profundas de la organización y funcionamiento de la economía y en el sistema de relaciones laborales. Además: "Se desarma la antigua regulación estatal, liberando y desregulando articuladamente las variables económicas de corto plazo y las instituciones y normas de funcionamiento con las que el Estado había intervenido tradicionalmente en la economía y en el sistema productivo argentino" (Catalano y Novick, 1996, p. 279).

En este marco, la ley de empleo sancionada en 1991 incorpora mecanismos de desregulación de las condiciones de contratación del desempeño de funciones y de estabilidad de los trabajadores.

Así mismo, aparecen los empleos por plazo determinado o indeterminado. Promocionándose contratos de formación - empleo, el trabajo para jóvenes, etc-. Previendo un sistema de prestaciones por desempleo, mientras gana un rol importante el Estado en materia de formación y reconversión profesional (Catalano y Novick, 1996).

A su vez, el decreto 1334 del mismo año, sujetó los incrementos salariales a la productividad, dificultándose el desarrollo de las negociaciones colectivas. Con esto se han incrementado los márgenes de las empresas de arbitrariedad debido a la individualización de la relación salarial. (Garro, 2010). 
Finalmente, mencionar junto a Garro (2010) que en este mismo periodo:

Se produjo la privatización parcial del sistema de jubilaciones y pensiones, el congelamiento del salario mínimo, la reforma de la Ley de accidentes de Trabajo, la desregulación parcial de las obras sociales sindicales, la habilitación vía legislación de modalidades contractuales atípicas, reducción de indemnizaciones, estímulo a la negociación por empresa, aumento de período de prueba entre otras reformas (p. 127).

\section{Flexibilización, precarización, desocupación e identidad}

Ante este panorama, una de las ideas que predominó la escena durante la década de los noventa sostenía que se arribaba "al fin de la sociedad del trabajo". Frente a dicha idea, Ruth Sosa (1999) dirá que lo que ha perdido centralidad es una forma específica de ejercer el trabajo, aquella que perfilaba una determinada identidad a todo trabajador o trabajadora:

Lo que hoy sin dudas pierde centralidad es aquella forma de ejercer el trabajo asociada a la categoría de "formal" y "estable", en la medida que hoy se "formaliza" lo que antes era "precario". La pérdida de esta referencia, asociada a una forma específica de ejercer el trabajo, nos desestructura y nos lleva a una crisis de identidad (p. 7).

Como se puede observar: la flexibilización, precarización y desocupación son fenómenos que el carácter de su hegemonía en el período analizado posibilita considerarlos como novedosos.

En relación a la flexibilización, Richard Sennett (2000) expresará:

En la actualidad, la expresión "capitalismo flexible" describe un sistema que es algo más que una mera variación sobre un viejo tema. El acento se pone en la flexibilidad y se atacan las formas rígidas de la burocracia y los males de la rutina ciega. A los trabajadores se les pide un comportamiento ágil; se les pide también -con muy poca antelación- que estén abiertos al cambio, que asuman un riesgo tras otro, que dependan cada vez menos de los reglamentos y de los procedimientos formales [...] El capitalismo flexible ha bloqueado el camino recto de la carrera, desviando repentinamente a los empleados de un tipo de trabajo a otro [...] La flexibilidad es el nombre que se usa para suavizar la opresión que ejerce el capitalismo (p. 3).

Algunos autores plantean una característica dual en el fenómeno de la flexibilización, a la vez que se esgrime del ataque a la burocracia y la rutina (Sennett, 2000), propiciando espacios para la creatividad y la iniciativa; produce por otra parte nuevas estructuras de poder y control bajo la lógica de la renta- 
bilidad empresarial. Al mismo tiempo incluyen otro componente radicalmente negativo: la precarización del empleo (Barbero, 2008).

Recuperando este planteo, Castel (1995) diría que la precarización daría origen a una nueva cuestión social regida por las nuevas exigencias tecnológicas - económicas de la evolución del capitalismo moderno, y la relaciona con el pauperismo en la primera mitad del siglo XX.

Es decir, lo que el fenómeno de precarización viene a instalar es la labilidad de la duración de los contratos de trabajo, como así también la abolición (no legal pero si real) de las prestaciones salariales en salud, pensión, educación, vacaciones, etc, cuyo cumplimiento y ejercicio fueron los patrones de lo que Ruth Sosa (1999) llamó "formal" y "estable".

Si el título de este apartado, además de los fenómenos de la flexibilización y precarización incluye el de la desocupación se debe a que el mismo es una consecuencia inexorable de un modelo de acumulación basado en el área de los servicios en detrimento de un modelo industrialista, el cuál requiere para su funcionamiento una cantidad de trabajadores mucho mayor que el que requiere el modelo económico hegemónico en el período analizado.

En este marco, y retomando las ideas iniciales tendientes a pensar la problemática de las identidades de los trabajadores y las trabajadoras en un contexto caracterizado por los fenómenos de la flexibilización, precarización y desocupación, Gabriel D’lorio (2005: 5), recuperando los planteos de Richard Sennett, sostiene en relación al status del trabajo, que "[...] la flexibilidad es la palabra esencial para pensar la experiencia contemporánea de la precariedad expresada en el fin de toda experiencia sólida y previsible". Aspectos éstos que a nivel de la subjetividad de los trabajadores se expresa en lo que Sennet (2000: 27) va a llamar corrosión del carácter, entendiendo a dicho fenómeno "[...] como efecto que quita al sujeto la orientación que hace posible la idea de carrera y de objetivos a largo plazo"; alimentando la doble sensación de una pérdida de identidad y una incertidumbre creciente sobre el futuro.

De esta manera, y a partir de lo planteado hasta el momento, si entendemos al trabajo como soporte privilegiado de inscripción en la estructura social, donde existen fuertes correlaciones entre el lugar que se ocupa en la división social del trabajo y la participación en las redes de la sociabilidad y en los sistemas de protección que cubren a un individuo ante los riesgos de la existencia, donde "[...] la asociación trabajo estable /inserción relacional sólida caracteriza una zona de integración [...], la ausencia de participación en alguna actividad productiva y el aislamiento relacional conjugan sus efectos negativos para producir la exclusión, o mas bien [...] la desafiliación", siguiendo con Castel "[...] la precarización del empleo y el aumento del desempleo constituyen sin dudas la manifestación de un déficit de lugares ocupacionales en la estructura social " Castel (1995: 15 - 416). 
En este contexto, frente a esta realidad es que la tradicionales identidades de trabajadoras y trabajadores han sido truncadas, y resignificadas en el mejor de los casos bajo parámetros desconcertantes para una subjetividad que anclaba sus andamiajes en un andar colectivo, en un relato donde el pleno empleo y las carreras lineales constituían los mayores sustentos de sentido.

\section{El lugar de la escuela y los jóvenes}

Pensar la construcción de sentidos sobre la escuela desde la conformación de un determinado mercado laboral implica pensar el trabajo como un soporte privilegiado de inscripción en la estructura social y no como mera relación técnica de producción (Castel, 1996).

Podemos observar como los distintos fenómenos ocurridos en el plano laboral tienen su repercusión en las demás instancias que hacen a la organización social.

En este punto, podemos hipotetizar una gran influencia de los procesos de flexibilización laboral en las distintas trayectorias de vida de los sujetos por distintas instituciones entre las cuales se incluye la escuela.

Por otra parte, los altos grados de desocupación han generado fuertes efectos que en concordancia con la crisis de identidad relatada anteriormente hacen emerger prácticas consideradas condicionadas y condicionantes de la valoración realizada, en este caso, sobre la escuela media.

En relación a nuestro universo de indagación más especifico, Marcelo Urresti (2005) reflexiona sobre lo significativo del desempleo para los jóvenes, ya sea por la escasez de recursos materiales como por el peso de encontrarse en un mundo social en el que la reproducción laboral no está garantizada:

El desempleo actual es altamente significativo para los jóvenes, que conviven con su escasez y sin el peso de su historia anterior, siendo ajenos a aquella cultura tradicional del trabajo y sus valores, percibiéndose en un mundo social en el que la reproducción laboral no está garantizada [...]La movilidad social descendente deja de ser una probabilidad para volverse palpable [...] Sin la incorporación social de los canales laborales, tambalea la función habitual cumplida por la escuela: la de ser entre otras cosas una instancia que prepara y capacita para una mejor inserción laboral y un eventual ascenso social (Urresti, 2005: 2).

Por otra parte, si pensamos que la institución escolar ocupaba un determinado lugar en estas trayectorias vitales de características lineales, habrían condicionado fuertemente las motivaciones de los jóvenes por el estudio y el trabajo (Jacinto, 2010). 
Se podría decir, según las propuestas esbozadas, que la escuela se inscribiría junto a demás instituciones propias de la modernidad en una "crisis" de sentidos, en la que las mismas pierden cierto lugar de "incuestionables".

Duschatzky, Silvia y Corea Cristina (2002) mencionan una pérdida de la autoridad simbólica e impotencia institucional por parte de las instituciones que durante los siglos XIX y XX orientaban el devenir de las personas. Mientras que Marcelo Urresti (2008) esboza:

[...] está aceptado que el conjunto de valores tradicionalmente implícitos que hicieron de la experiencia escolar una preparación para el trabajo, esa cultura común basada en el esfuerzo, el sacrificio y la inversión, pasan por una reestructuración de la que no se sabe todavía sus consecuencias, aunque haya acuerdos sobre el debilitamiento de su validez (p. 62).

Si bien los hechos que desencadenan en las mutaciones de sentidos vividas por todas las instituciones de la sociedad deben ser inscriptas en las mutaciones orgánicas del sistema capitalista y a los intereses en funciones de toma de decisiones como gran protagonistas de las características que presentará una sociedad en determinados momentos, en este caso se nos hace imposible negar el lazo más intrínseco y claro entre el mercado laboral y la escuela, como institución que entre otros fines ha respondido históricamente a la demanda de ascenso social.

En el siguiente apartado observaremos ciertos cambios en las características que configuraron el mercado laboral en el período hasta aquí analizado, este análisis induciría a una revisión de los planteos recientemente esbozados sobre los sentidos construidos por los jóvenes en relación a la escuela media. Poniendo en cuestión, de esta manera, ciertas ideas que han predominado la escena educativa hasta el momento.

\section{Reconfiguración del mercado laboral ¿Barniz o una configuración posible?}

Para pensar la nueva configuración del mercado laboral, nos hemos apoyado fundamentalmente en el trabajo dirigido por Héctor Palomino Las nuevas dinámicas de la relaciones laborales en Argentina.

Uno de los diagnósticos aventurados a partir de estos estudios, llevan a Palomino a expresar que entre los años 2003 y 2008, que es el período en que se llevan a cabo las investigaciones realizadas:

[...] Se registra la configuración de un nuevo régimen de empleo que, junto con el fortalecimiento de las instituciones laborales, generó un marco diferente para las relaciones de trabajo en Argentina. En este marco se observan el afianzamiento del rol del Estado, un 
protagonismo recobrado de los sindicatos y una canalización de las demandas salariales a través de la negociación colectiva, fenómenos que contrastan puntualmente con los registrados en la década anterior. Pero también se observan, en contrapunto con aquellas tendencias, la consolidación de tendencias previas, algunas antiguas, otras más recientes (Palomino, 2010: 17).

Como podemos observar, el fenómeno no puede ser generalizado ni analizado desde miradas duales, a la vez que tampoco debe ser analizada la configuración del mercado laboral desde una perspectiva sincrónica. Es decir, pensar un período implica tener en cuenta la complejidad de un proceso.

Ejemplo de ello son ciertas conclusiones a las que se arriban a partir de un estudio teórico sobre las distintas perspectivas desde dónde y cómo pensar la informalidad del mercado laboral, realizadas por una de las integrantes del equipo dirigido por Palomino. En las mismas su autora expresará que a pesar de las tendencias positivas mencionadas, investigaciones actuales a partir de distintos abordajes teóricos y metodológicos muestran la persistencia de distintos trabajos informales y / o asalariados con condición de precariedad, considerando desde un carácter estructural el problema de la informalidad en el mercado laboral de nuestro país (5). En la misma línea, a partir del análisis de algunos rubros del sector alimenticio se concluye que la precarización del mercado laboral no ha retrocedido y se continúa con una tendencia ya existente. El mismo propone analizar el caso argentino en el marco de una tendencia a nivel global donde "las estrategias empresariales de subcontratación, de externalización y de flexibilización siguen contribuyendo a fragmentar y a precarizar los mercados laborales" (Senén González, 2010, p. 154).

Sin embargo, por otra parte existen una serie estudios que tratan de poner la lupa en aspectos más auspiciosos de la actual configuración del mercado laboral.

Por ejemplo, sin desconocer la existencia de situaciones de informalidad y precariedad, Palomino (2010) saca a lucir algunas estadísticas que reflejarían un panorama no tan trágico para la situación de los trabajadores de nuestro país.

En relación a la evolución del trabajo no registrado, en base a datos del INDEC (Instituto Nacional de estadísticas y Censo) [6], nos muestra que entre el IV trimestre del 2004 y el I del 2007, los asalariados registrados aumentaron en 1.265.000 ocupados, mientras que los asalariados no registrados disminuyeron en 402.000 ocupados (Palomino, 2010). Ubicando esta tendencia como corte a un proceso continuo existente desde hace por lo menos dos décadas.

Siguiendo con el análisis procesual, a la hora de analizar el desarrollo del comportamiento de los trabajadores como sector, Palomino observa en los últimos años un impulso a la negociación colectiva como tendencia. Va a decir que mientras en los años '90 se registró un promedio de 200 negociaciones colectivas homologadas anualmente, en el 2007, que el año que alcanza su 
estudio, se registraron 1027 negociaciones homologadas. Datos más actuales muestran que mientras en el año 2004 existieron 348 negociaciones que incluían un total de 1.222.000 trabajadores, en el año 2010 se llevaron a cabo 2038 negociaciones que incluían 4.220.161, y en 2011, si bien las negociaciones pasaron a ser 1864, la cantidad de trabajadores incluidos alcanzaba la cifra de 4.235.000 (Zaiat, 2012).

Lo que a primera vista expresaría un fortalecimiento sindical que articulado con los datos de la disminución de la desocupación y la subocupación mostraría un aumento de los trabajadores inscriptos y a la vez sindicalizados.

Por otra parte, atendiendo a esta nueva situación del mercado laboral, el Estado es considerado como actor fundamental en las tendencias actuales, sobre todo por la recuperación de su capacidad de arbitraje a partir de su rol mediador de intereses contrapuestos. En este marco es que Palomino (2010) va a hablar de un nuevo régimen laboral. Expresará:

La idea de régimen apunta a la determinación de una serie de complementariedades institucionales, que vinculan la recuperación del rol de arbitraje de control del Estado sobre el registro laboral, con la reinstalación normativa del control jurídico sobre la subcontratación, con las políticas públicas del salario mínimo y la negociación colectiva y, finalmente, con el cambio de comportamiento de las organizaciones sindicales, los trabajadores y otros actores sociales (p. 204).

En esta línea, un caso curioso que señala Palomino (2010) tiene que ver con la jurisprudencia asentada en hechos de conflictos laborales. En una clara muestra de los lineamientos de las acciones estatales, mencionará que para ejecutar los cambios en las tendencias a disminuir las prácticas de precarización, no se ha recurrido a una modificación del cuerpo legal del régimen de empleo sino a su interpretación a través de la jurisprudencia respectiva.

Ahora bien, si hasta el momento podemos intuir que las tendencias que configuran el mapa del mercado laboral actual en nuestro país se orientan a generar fisuras con aquel que predominó la escena en la década del los '90, no debemos contentarnos como si nos acercaríamos al ideal ni mucho menos.

Bien va a decir Palomino (2010) a partir de la pregunta por los límites de los mecanismos laborales descriptos, que la política laboral actual no está orientada a instituir una regulación global, mas bien regulaciones específicas en determinados sectores y actividades. Es decir, si bien se impusieron en este período medidas estructurales con sentido protectorio en más de una década, otras fueron más de índole coyuntural.

De esta manera, y como se menciona al comienzo del apartado, la configuración del mercado laboral en la actualidad no debe ser pensado de forma dual ni mucho menos. Por el contrario, y considerando las políticas laborales en el marco de procesos políticos más amplios que incluyen toma de decisiones 
en situaciones contradictorias y conflictivas, podemos sugerir que el panorama descripto, sin dejar de ser preocupante, introduce algunos elementos que pueden ser leídos como indicios de un quiebre con el patrón que se impuso con carácter hegemónico en la década de los '90. Y con ello lógicas diferentes de acción y movimiento que irían generando nuevas significaciones. Que si bien aún no se puede aseverar lo auspicioso de las mismas revisan ciertas situaciones con carácter de criticidad.

\section{Conclusiones}

Con el fin de rastrear aspectos que den cuenta del proceso de construcción de sentidos que los jóvenes le dan a la escuela media en el paso por sus vidas, se ha realizado un análisis comparativo a nivel socio-histórico en el contexto argentino, en pos de buscar una potencial relación entre la construcción de sentido de la escuela media y los cambios experimentados por el mercado laboral. Esto es, comenzamos a describir una realidad social donde el trabajo "formal" y "estable" ha dejado de ser el eje organizador de la vida de los sujetos en la década de los 90", y como la escuela se ubica ante esa realidad en la representación que los jóvenes tienen de ella. Hasta llegar a la observación de una emergente reconfiguración del mercado laboral en la actualidad. Este ejercicio más que brindarnos certezas, abrió más incógnitas, sin dejar de enriquecer la complejidad que deriva del pensar la construcción de sentidos sobre la escuela media.

En pos de realizar un cierre y resumen del recorrido desandado en el presente artículo, mencionamos que este consistió en cinco momentos y/o apartados. El primero, refiere a una mención de carácter histórica sobre la construcción de sentidos sobre la escuela como institución en un marco nacional, resulta imprescindible mencionar este camino que ha ido configurando a la escuela como una institución de autoridad indefectible dentro de la sociedad argentina. Y con esto, la importancia que adquiere el rol del Estado en tanto donante y creador de sentidos, que van permeando dicha institución. Vimos en el mismo, la eficacia simbólica que se va ejerciendo en esta relación escuela y estado, ascenso social mediante, con el fin de introducirnos en la complejidad que requiere pensar los elementos que fueron caldeando nuestro tema de análisis.

En un segundo momento, se propuso una descripción del mercado laboral de los 90', entendiendo que el capitalismo atraviesa a lo largo de toda su historia una serie de procesos de cambio y ruptura tanto cuali como cuantitativos de tipo estructural. Asimismo, estos procesos son permanentes y cíclicos, y en este devenir van involucrando a todas las esferas de práctica social, por ejemplo, la institución escuela. Caracterizamos a la morfología del mercado laboral de los 90', como un proceso que comienza en la década de los 70', en esta época se convierte en un elemento central la valorización de la forma 
financiera de capital, alterando todas las formas institucionales de la sociedad, y las relaciones sociales en ellas comprendidas. Observamos como esos cambios que se vienen gestando desde aquellos años, fueron dándole forma en los '90 a una fuerza de trabajo flexible para la actividad económica-productiva. Esta flexibilidad, fue moldeada en la medida en que se fue requiriendo de una mayor disponibilidad de trabajo de carácter rotativo, relocalizado, capacitado o disponible para eventuales reemplazos. Incorporando mecanismos de desregulación de las condiciones de contratación del desempeño de funciones y de estabilidad de los trabajadores. En este análisis también se incluyó, el desarme que ha experimentado la antigua regulación estatal.

Un tercer momento nos llevó a repensar la relación entre flexibilización, precarización y desocupación emergentes de la morfología del mercado laboral de los 90' y los procesos de construcción de identidades tanto colectivas como individuales. Se entiende que estas características sobresalientes del mercado laboral de los 90', fue imprimiendo en los sujetos una doble sensación, por una lado la pérdida de identidad y por otro una creciente incertidumbre sobre el futuro. De este modo, se observa el fin de proyectar una vida a partir de un determinado trabajo y como la nueva lógica capitalista exige una reconversión continua de uno mismo, lo cual deriva en una crisis de las identidades tradicionales. Esta nueva realidad fue re-significando nuevas subjetividades posibles, y en este punto se encuentra insoslayable la relación existente entre la construcción de sentidos sobre la escuela y la conformación de un determinado mercado laboral, a partir de ver a la escuela como parte de un engranaje social más complejo.

El cuarto apartado, nos vio inmersos en la cuestión de la escuela y los jóvenes a partir de analizar la gran influencia de los procesos de flexibilización laboral en las distintas trayectorias de vida de los sujetos. Enmarcamos a la escuela como una institución más dentro de la estructura social, y observamos que la ruptura de la linealidad de trayectorias vitales producida fundamentalmente por las mutaciones del mercado laboral condicionaron fuertemente las motivaciones de los jóvenes por el estudio y el trabajo. Sobre todo si pensamos en el lazo más intrínseco y claro entre el mercado laboral y la escuela, como institución que entre otros fines ha respondido históricamente a la demanda de ascenso social.

Finalmente, en un quinto momento, arribamos a la cuestión de repensar las características actuales del mercado laboral argentino, en pos de no caer en la falacia de no reconocer cortes y movimientos históricos en el mismo, y a partir de allí comenzar a pensar cómo se pueden ir redefiniendo los sentidos de los jóvenes en relación a la escuela media con una morfología diferente del mercado laboral. En este punto, se registra que la configuración de un nuevo régimen de empleo junto con el fortalecimiento de las instituciones laborales, generó un panorama diferente para las relaciones de trabajo en Argentina. En este marco se observan el afianzamiento del rol del Estado, un protagonismo 
recobrado de los sindicatos y una canalización de las demandas salariales a través de la negociación colectiva, fenómenos que contrastan puntualmente con los registrados en la década anterior. Sin embargo, se observan simultáneamente la consolidación de tendencias previas.

De esta manera, el presente trabajo intentó dar un elemento más de análisis para entender la construcción de sentidos de los jóvenes en torno a la escuela media, pero además, se apuntó a repensar este nuevo contexto socio histórico específicamente a partir de la descripción del mercado laboral como un emergente de nuevos significados y lógicas a partir de los entrelazamientos de prácticas conjuntas. Es decir, al poner nuestra mirada sobre el mercado laboral, entendemos a este como un canal más de expresión de lo que una sociedad, en este caso la argentina nos está mostrando. A partir de esta perspectiva, quisimos poner en cuestión una nueva reconfiguración de este, para abrir el entendimiento hacia nuevas construcciones de sentidos posibles, entendiendo a la institución escuela, y los miembros de estas, inmersos en un contexto social amplio, y entrechocándose con otras esferas de lo social en esta conformación simbólica de representaciones.

Por lo tanto, han surgido a partir de este estudio nuevas preguntas, ¿si es que se ha generado una reconfiguración del mercado laboral argentino, que nuevos sentidos se fueron dibujando en torno a la escuela? ¿Esos sentidos han ido cambiando? ¿Cuales siguen muy vigentes?

Entendemos que el quiebre de la linealidad de las trayectorias de vida sea una característica que exceda la morfología del mercado laboral, la misma ha impregnado en la lógica de las subjetividades de los jóvenes y adolescentes de nuestro país. En este sentido, si bien entendemos y compartimos la substancialidad del peso del trabajo en la organización de una sociedad, tal vez han sido diversos y múltiples los motivos enlazados que provocaron el surgimiento de nuevas trayectorias. De esta manera, si bien las mismas dejaron de representar una linealidad que descansaba en las certezas del recorrido, si descansen en la posibilidad de visualizar ciertas opciones, entre las cuales se encuentre la de habitar la escuela.

En este mismo sentido, y este es uno de los desafíos que restan recorrer, este deseo o elección de un determinado orden (habitar una escuela) no debemos esperar encontrarlo en los formatos que impregnaron históricamente nuestro paisaje. Las trayectorias no lineales adquirieron vida propia, es desafío reflexionar sobre las lógicas y los sentidos que orientan a las mismas. En eso estamos. 


\section{Notas bibliográficas}

(1) El en presente artículo se propone reelaborar y profundizar el trabajo llevado a cabo por uno de los autores del mismo, en el marco elaboración de tesis Doctoral en Educación De la Facultad de Ciencias de la Educación de la Universidad Nacional de Entre Ríos.

(2) Siguiendo ideas de Laplanche y Pontalis (1971).

(3) Fuente: INDEC, EPH continua.

(4) Entendemos la eficacia simbólica como un poder vinculado a la facultad del convencimiento que reviste el discurso (Bourdieu, 1999: 71).

(5) Un estudio realizado por el Ministerio de Trabajo y Seguridad Social de la Nación en el 2007 muestra que para el primer trimestre del año 2005, el 43\% de los 5.300 .000 de trabajadores que residían en el gran Buenos Aires se encontraban en condiciones de informalidad.

(6) Encuesta Permanente de Hogares de INDEC. Realizada por Diego Schlesser y colaboradores de la Dirección General de Estadísticas y Estudios Laborales de la SSPTyEL, para el Boletín de Estadísticas Laborales del MTESS.

\section{Referencias bibliográficas}

- Barbero, J. (2008). Reconfiguraciones de la comunicación entre escuela y sociedad. En Tenti Fanfani (comp.); Nuevos temas en la agenda de política educativa (pp. 65 - 99). Buenos Aires: Ed. Siglo XXI.

- Braslavsky, C. (1995). La discriminación educativa en Argentina. Bs. As.: Ed. Miño y Dávila.

- $\quad$ Carbonari, F. (2009). Limites y posibilidades de constitución como profesionales críticos y transformadores en la Universidad Pública hoy. Cuarto Encuentro Nacional de Educación. 11, 12 y 13 de septiembre de 2009. Rosario. Argentina

- $\quad$ Castel, R. (1995). La metamorfosis de la cuestión social: una crónica del salariado. Bs. As. : Ed. Paidós.

- $\quad$ Castillo, V. (2006). La Extensión Universitaria. En el Marco de la Educación Popular: Una Lectura Desde Gramsci. Núcleo Socio- Educativo, Carrera de Ciencias de la Educación, Facultad de Humanidades y Artes, UNR. Rosario, Argentina.

- $\quad$ Catalano, Ana M. y Novick, M (1996). La Sociología del Trabajo al encuentro de las relaciones laborales en un marco de incertidumbre. En Marta Panaia (comp.) Trabajo y empleo. Un abordaje interdisciplinario (pp. 249 - 298). Bs. As. : Coediciones Eudeba y Paite.

- D`lorio, G. (Abril, 2005). Sentidos y problemas en torno a lo precario. Apuntes para una crítica de sus formar contemporáneas. Ponencia presentada en Seminario Internacional: La escuela hoy. Desafíos, Debates, Perspectivas. Huerta Grande, Córdoba, Argentina.

- Duschatzky, S y Corea, C (2002). Chicos en banda. Los caminos de la subjetividad en el declive de las instituciones. Bs. As: Ed. Paidós.

- Duschatzky, S. (1999). La escuela como frontera. Reflexiones sobre la experiencia escolar de jóvenes de sectores populares. Bs. As.: Ed. Paidós.

- Dubet, F., (2006). El Declive de la institución: Profesiones, sujetos e individuos ante la reforma del Estado. Barcelona: Ed. Gedisa.

- Gentili, P. (1997). Adiós a la escuela pública. El desorden neoliberal, la violencia del mercado y el destino de la educación de las mayorías. En, Gentili, P. (comp.); Cultura, política y currículo. Ensayos sobre la crisis de la escuela pública (pp. 111 - 144). Bs. As. : Ed. Losada. 
- Jacinto, C. (2010) (Compiladora). La construcción social de las trayectorias laborales de jóvenes. Políticas, instituciones, dispositivos y subjetividades. Programa de Estudios sobre Juventud, Educación y Trabajo (PREJET-IDES).

- Jacinto, C. (2011). La educación dejó de ser algo que se adquiere en un momento determinado de la vida. Nota realizada por el diario Página 12. Lunes 9 de mayo de 2011.

- Palomino, H. (2010). Las nuevas dinámicas de las relaciones laborales en Argentina. Presentación. Bs. As.: Ed. Jorge Baudino Ediciones..

- Palomino, H. (2010). La instalación de un nuevo régimen de empleo en la Argentina: De la precarización a la regulación. En Palomino, H. (comp.); Las nuevas dinámicas de las relaciones laborales en Argentina (pp. 179 - 206). Bs. As.: Ed. Jorge Baudino Ediciones.

- Pogliaghi, L. (2010). La informalidad en el marco de las relaciones de tabajo: Importancia de su estudio y perspectivas teórico - analíticas para su abordaje. En Palomino, $\mathrm{H}$. (comp.); Las nuevas dinámicas de las relaciones laborales en Argentina (97 - 122). Bs. As.: Ed. Jorge Baudino.

- Senén González, C. (2010). Tendencias recientes de las relaciones laborales en la Argentina: Fortalecimiento y precarización en el sector de alimentación. En Palomino, $\mathrm{H}$. (comp.); Las nuevas dinámicas de las relaciones laborales en Argentina (pp. 153 - 178). Bs. As. : Ed. Jorge Baudino Ediciones.

- Sennett, R. (2005). La corrosión del carácter. Consecuencias personales del trabajo en el nuevo capitalismo. La Habana. Cuba: Ed. De Ciencias Sociales.

- Sosa, R. (1999); ¿Crisis de la sociedad del trabajo?... ¿O nuevo sentido común posmoderno? En, Taborda, M. (1999 / 2000); Anuario de Sociología (pp. 7 - 21). Departamento de Sociología. Facultad de Ciencias Políticas y Relaciones Internacionales. Universidad Nacional de Rosario (U.N.R.). Rosario. Argentina: Ed. UNR editora.

- Tedesco, J. C. (2005). Educación popular hoy: ideas para superar la crisis. En Nun J. (Director); Claves para todos (pp. 13, 14). Bs. As.: Ed. Capital intelectual.

- Tenti Fanfani, E (2002). La educación media en la Argentina: desafíos de la universalización. En Tenti Fanfani, E. (comp.). Educación media en Argentina. Desafíos de la universalización (pp. 11 -33). Bs. As.: IIPE - UNESCO, Altamira y Fundación OSDE.

- Tiramonti, G (2005). La fragmentación educativa y los cambios en los factores de estratificación. En Tiramonti (Comp.). La trama de la desigualdad educativa. Mutaciones recientes en la escuela media (pp. 5 - 24). Bs. As.: Ed. Manantial.

- Urresti, M. (2005). Adolescentes en la encrucijada. La tensión entre la escuela y las brechas sociales, comunicativas y culturales. Ponencia presentada en Seminario Internacional: La escuela hoy. Desafíos, Debates, Perspectivas. Huerta Grande, Córdoba, Argentina.

- Urresti, M. (2008). Nuevos procesos culturales, subjetividades adolescentes emergentes y experiencia escolar. En Tenti Fanfani (comp.) Nuevos temas en la agenda de política educativa (pp. 100 - 124). Bs . As.: Editorial Siglo XXI.

- Vizcarra Fernando (2002). Premisas y conceptos básicos en la Sociología de Pierre Bourdieu. En Estudios sobre Culturas Contemporáneas. Volumen VIII. Número 16. Universidad de Colima. Colima, México. Disponible en: <http://redalyc.uaemex.mx/redalyc/ src/inicio/ArtPdfRed.jsp?iCve=31601604> ISSN 1405-2210.

- Zaiat, A. (2012). Salario Real. Nota publicada en el diario Página 12 el Domingo, 15 de julio de 2012. 